\title{
Syllogistic reasoning time: Disconfirmation disconfirmed
}

\author{
VALERIE A. THOMPSON, CHRISTOPHER L. STRIEMER, RHETT REIKOFF, \\ RAYMOND W. GUNTER, and JAMIE I. D. CAMPBELL \\ University of Saskatchewan, Saskatoon, Saskatchewan, Canada
}

\begin{abstract}
Models of deductive reasoning typically assume that reasoners dedicate more logical analysis to unbelievable conclusions than to believable ones (e.g., Evans, Newstead, Allen, \& Pollard, 1994; Newstead, Pollard, Evans, \& Allen, 1992). When the conclusion is believable, reasoners are assumed to accept it without much further thought, but when it is unbelievable, they are assumed to analyze the conclusion, presumably in an attempt to disconfirm it. This disconfirmation hypothesis leads to two predictions, which were tested in the present experiment: Reasoners should take longer to reason about problems leading to unbelievable conclusions, and reasoners should consider more models or representations of premise information for unbelievable conclusions than for believable ones. Neither prediction was supported by our data. Indeed, we observed that reasoners took significantly longer to reason about believable conclusions than about unbelievable ones and generated the same number of representations regardless of the believability of the premises. We propose a model, based on a modified version of verbal reasoning theory (Polk \& Newell, 1995), that does not depend on the disconfirmation assumption.
\end{abstract}

One widely replicated phenomenon in reasoning research is the belief bias effect: Reasoners accept a conclusion or argument more readily when it accords with their beliefs than when it does not, regardless of the validity of the conclusion or the soundness of the argument (e.g., Baron, 1995; Edwards \& Smith, 1996; Evans, Barston, \& Pollard, 1983; Evans, Newstead, Allen, \& Pollard, 1994; Koehler, 1993; Newstead, Pollard, Evans, \& Allen, 1992; Stanovich \& West, 1997; Thompson, 1996). A widely accepted explanation for this effect is the disconfirmation hypothesis, which states that reasoners are more critical of conclusions and arguments that they do not believe and are more likely to search for reasons to refute or disconfirm an unbelievable conclusion than a believable one (Edwards \& Smith, 1996; Evans, Newstead, \& Byrne, 1993; Koehler, 1993; Newstead et al., 1992). A logical extension of this hypothesis is that it should take reasoners longer to reason about unbelievable conclusions (Edwards \& Smith, 1996), because they spend more time analyzing situations that contradict their beliefs, relative to situations that confirm their beliefs.

The goal of the present paper is to test this hypothesis in the context of deductive reasoning. There is a large literature dealing with factors that determine the acceptability of conclusions, but not with the amount of time required to process conclusions. Thus, in the present paper,

This research was supported by an operating grant from the Natural Sciences and Engineering Research Council of Canada. Address correspondence to V. A. Thompson, Department of Psychology, University of Saskatchewan, 9 Campus Drive, Saskatoon, SK, S7N 5A5 Canada (e-mail: valerie.thompson@usask.ca). we measured the time required to evaluate arguments leading to believable and unbelievable conclusions.

\section{Disconfirmation and Deductive Reasoning}

The literature reveals three robust effects associated with the evaluation of deductive arguments (e.g., Evans et al., 1994; Evans et al., 1993; Klauer, Musch, \& Naumer, 2000; Thompson, 1996). As was stated earlier, believable conclusions are accepted more often than unbelievable ones. Reasoners do not base their judgments solely on their beliefs; they also accept a greater number of valid than invalid conclusions. There is also a large interaction between validity and believability, such that the effect of validity (i.e., the difference in the rate of acceptance for valid and invalid conclusions) is larger for unbelievable conclusions than for believable ones.

The disconfirmation hypothesis offers a compelling explanation for this pattern (Evans et al., 1994; Evans et al., 1993; Newstead et al., 1992). When the conclusion is believable, reasoners are inclined to accept it without further scrutiny. When it is unbelievable, they are more likely to engage in a logical analysis of the problem. This analysis allows them to discriminate between valid and invalid conclusions, such that the effect of validity is larger for unbelievable conclusions than for believable ones.

\section{Mental Models Theory}

The mental models theory of deductive reasoning (hereafter, MMT) offers the most widely accepted version of the disconfirmation hypothesis (Johnson-Laird \& Byrne, 1991; Newstead et al., 1992; Oakhill \& Johnson-Laird, 1985; Oakhill, Johnson-Laird, \& Garnham, 1989). Ac- 
cording to MMT, deductive reasoning occurs in three stages. First, reasoners construct a mental model of the premise information. Second, they draw a conclusion that is consistent with that information, or in the case in which a conclusion is provided, they evaluate whether the conclusion is consistent with the model (Evans et al., 1993). Finally, they search for an alternative model that may falsify the initial conclusion. This last stage, which shall be referred to as the disconfirmation process, is effortful and demanding of mental resources, and reasoners may neglect this step unless specifically motivated to do otherwise. For example, it is assumed that disconfirmation is more likely to occur for unbelievable conclusions than for believable ones (e.g., Newstead et al., 1992; Oakhill et al., 1989), presumably because reasoners are seeking a means to refute an unpalatable conclusion.

Collectively, these assumptions explain the widely observed interaction between validity and belief: Reasoners are likely to search for a disconfirming model when presented with an unbelievable conclusion, but not when presented with a believable conclusion. When the conclusion is invalid, the search is successful, and the conclusion is rejected. In contrast, because all legitimate models are consistent with a valid conclusion, it will be accepted. Thus, for unbelievable conclusions, there is a large difference in the rate at which valid and invalid conclusions are accepted. In contrast, when the conclusion is believable, reasoners are unlikely to search for disconfirming models and, thus, do not discriminate between valid and invalid conclusions.

The goal of the present research was to provide a test of the disconfirmation hypothesis, using a response time (RT) analysis. We used a standard paradigm (Evans et al., 1983; Evans et al., 1994; Klauer et al., 2000; Newstead et al., 1992; Oakhill et al., 1989; Thompson, 1996) in which reasoners were presented with pairs of premises and then were asked to judge whether a given conclusion followed logically from those premises. The predictions based on the disconfirmation hypothesis are clear and straightforward: The more alternatives a reasoner considers, the longer the problem should take to solve. Thus, reasoners should take longer when presented with unbelievable conclusions than with believable ones, because reasoners are more likely to engage in a search for disconfirming models when presented with unbelievable conclusions.

Similarly, it should take reasoners longer to accept valid conclusions than to reject invalid ones: In order to reject an invalid conclusion, only a single, disconfirming model need be found, whereas to accept a valid conclusion, the reasoner must be satisfied that the conclusion is consistent with all possible models of the premises. Moreover, because disconfirmation is more likely to occur for unbelievable conclusions than for believable ones, it follows that an interaction between beliefs and validity should be observed for RTs as well as for rates of acceptance: The difference between the time required to process valid and invalid conclusions should be larger for unbelievable conclusions than for believable ones.
Finally, we implemented a second test of the disconfirmation hypothesis. Following each problem, reasoners were asked to draw as many diagrams as possible that were consistent with the premise information (Newstead, Handley, \& Buck, 1999; Newstead, Thompson, \& Handley, 2002; Torrens, Thompson, \& Cramer, 1999). Because it is assumed that reasoners are more likely to engage in a search for disconfirming models when they are presented with unbelievable conclusions relative to believable ones, it follows that more such diagrams should be generated when the conclusions are unbelievable.

\section{METHOD}

\section{Participants}

One hundred twenty-eight students from the University of Saskatchewan participated. Half participated in partial fulf illment of a course requirement; the remainder were paid a small sum (\$5). Seventy-three percent were female, and the mean age was 22.36 years $(S D=7.26)$.

\section{Materials}

The materials for both studies consisted of the three-term syllogisms listed in Table 1. For each problem, the participants were presented with two premises, followed by a conclusion of the form "Some _ are not _." Two sets of problem materials were developed. For Set 1, the problems were identical to those used by Evans et al. (1994, Experiment 1). These problems combined one premise of the type "No _ are __ "with one premise of the type "Some _ are _.." Set 2 materials were created by substituting a premise using "All" for the premise using "Some." Valid and invalid versions of each problem were created, as is illustrated in Table 1. For both sets of materials, there were two groups of problems, one leading to conclusions of the form "Some of the C are not A," and one leading to conclusions of the form "Some A are not C." We did not investigate differences among these two types of conclusions; they were included in order to control for well-known figural effects (e.g., Evans et al., 1993).

Content was assigned to the $\mathrm{A}, \mathrm{B}$, and $\mathrm{C}$ terms such that the $\mathrm{A}$ and $\mathrm{C}$ terms referred to familiar categories (e.g., well-educated people and judges) and the B term was a nonsense term (e.g., Pennes). This was done in order to avoid any effects that might be attributed to the believability of the premises (Thompson, 1996). Eight different A, B, and C terms were chosen for this study; each set of A,

Table 1

Logical Forms of the Problems Used in the Experiment

\begin{tabular}{lll}
\hline \multirow{2}{*}{ Version } & \multicolumn{2}{c}{ Problem Set } \\
\cline { 2 - 3 } Valid & \multicolumn{1}{c}{ "Some C are not A" Conclusions } \\
& No A are B & No B are A \\
& Some C are B & All B are C \\
& Therefore, some C are not A & Therefore, some C are not A \\
Invalid & Some A are B & All B are A \\
& No C are B & No C are B \\
& Therefore, some C are not A & Therefore, some C are not A \\
& \multicolumn{1}{c}{ "Some A are not C" Conclusions } \\
Valid & Some A are B & All B are A \\
& No C are B & No C are B \\
& Therefore, some A are not C & Therefore, some A are not C \\
Invalid & No A are B & No B are A \\
& Some C are B & All B are C \\
& Therefore, some A are not C & Therefore, some A are not C \\
\hline
\end{tabular}


$\mathrm{B}$, and $\mathrm{C}$ terms could be accompanied by both a believable conclusion (e.g., Some of the well-educated people are not judges) and an unbelievable conclusion (e.g., Some of the judges are not welleducated people); the believability of these conclusions was established in a rating study by Evans et al. (1983).

\section{Design and Procedure}

The participants received one block of "Some A are not C" conclusions and one block of "Some $\mathrm{C}$ are not A" conclusions; order was counterbalanced across participants. Each block consisted of one problem in each belief by validity cell; the order of these conditions was determined by a Latin square, which was repeated every 4 participants. Content was assigned to problems in such a way that no problem content was repeated during the eight problems, and each of the eight problem contents was assigned equally often to each of the experimental conditions.

There was one problem per page. Following each problem were two options, "yes" and "no," and a blank space to draw diagrams. Instructions were written and stapled to the front of the booklet; these were adapted from the standard instructions used by Evans et al. (1994). The instructions emphasized that the reasoners were to judge whether or not a conclusion followed logically from the premises; if they judged that a conclusion followed logically, they were instructed to say "yes"; if the conclusion did not necessarily follow from the premises, they were to say "no." The participants were instructed not to make notes or diagrams while solving the problems and not to return to previous problems after they had been completed.

Timing was done manually, using a stop watch accurate to $0.01 \mathrm{sec}$. There was a blank sheet on the top of the test booklet. The experimenter, who was blind to the belief and validity status of the conclusions, gave a verbal signal to begin and started the timer. The participant turned the page over; timing ended when the response was spoken aloud. After giving their responses, the participants were instructed to draw as many diagrams as possible that were consistent with the premises (see below). After the diagrams were completed, the experimenter gave the signal to begin the next trial.

In total, there were four between-subjects experimental conditions, which were run as two separate studies, as described below.

Study 1. There were two groups of participants: 32 participants completed both the reasoning and the diagram portion of the experiment, and another 32 completed only the reasoning portion. All the participants were tested with Set 1 materials, as described in the Materials section. For the diagrams, the participants were instructed to draw Euler circle representations that integrated the A, B, and C terms. They were given examples and two practice trials prior to beginning the experimental trials. The participants were given feedback after each practice trial.

Study 2. Thirty-two participants reasoned with Set 1 materials, and another 32 with Set 2 materials. All of the participants drew diagrams. However, instead of Euler circles, the participants were instructed in the use of standard mental models notation and were asked to represent the premise information in that form. In mental models notation, sets are represented by groups of tokens, and the relationship between sets is designated by the overlap between the groups. For example, the premise "Some of the A are B" would be represented as follows. This representation depicts a set of $A$ s, some of which overlap with a set of $B \mathrm{~s}$ :

$$
\begin{array}{rl}
a & a \\
a & =b \\
& b
\end{array}
$$

\section{RESULTS}

The patterns of acceptances, RTs, and diagrams produced were practically identical across the four groups of participants (Study 1, diagrams and no diagrams; Study 2, old and new materials), so the data from the four groups were combined and analyzed together in all of the analyses reported below. ${ }^{1}$ Figure 1 presents the mean proportion of conclusions accepted, RTs, and number of diagrams drawn as a function of conclusion validity and believability. The data were analyzed using $2 \times 2$ (validity $\times$ belief) within-subjects analyses of variance (ANOVAs) for each of the three dependent measures; for the conclusions accepted and RT analyses, $d f=1,127$; for the diagrams analysis, $d f=1,95$ for all tests.

\section{Analysis of Conclusions Accepted and RTs}

As the middle panel of Figure 1 illustrates, the data replicate the standard findings $(p<.001)$ regarding rates
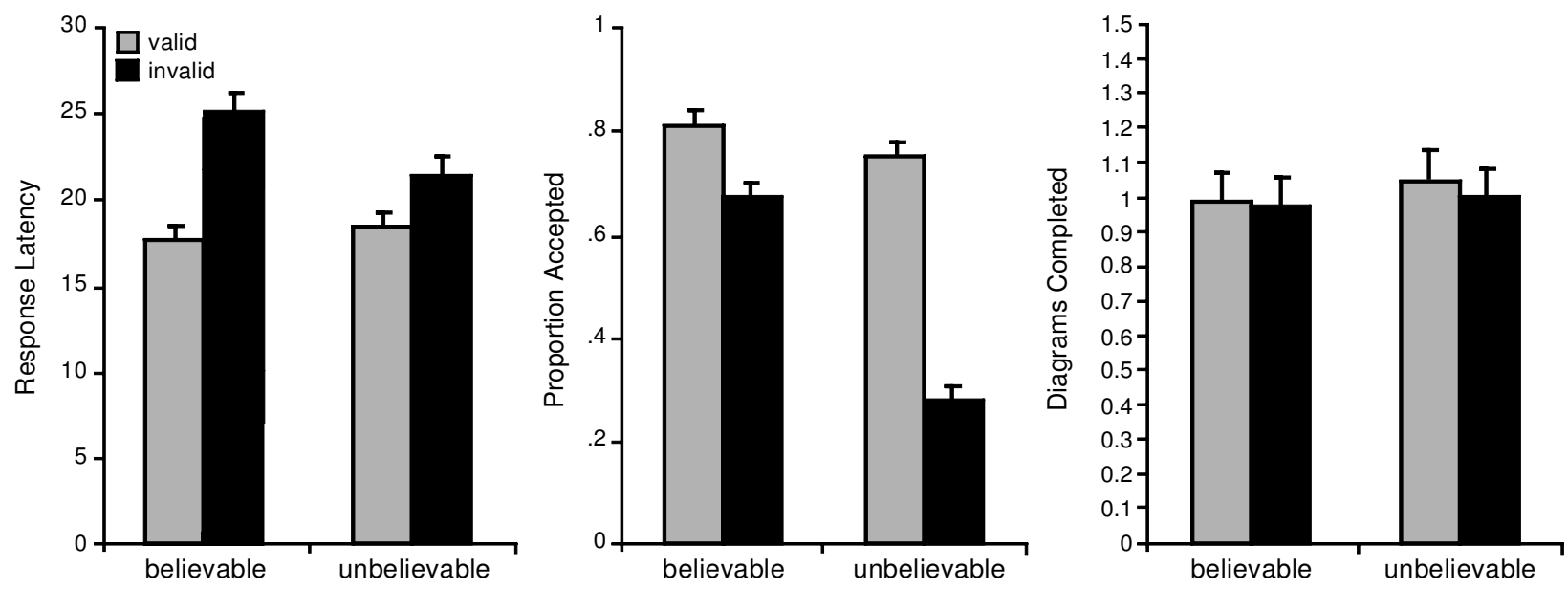

Figure 1. Response time, proportion of conclusions accepted, and number of diagrams completed as a function of conclusion believability and validity. 
of acceptance (Evans et al., 1983; Evans et al., 1994; Newstead et al., 1992; Thompson, 1996): The reasoners accepted more valid (.77) than invalid (.47) conclusions $\left(F=94.1, M S_{\mathrm{e}}=0.13\right)$ and accepted more believable (.74) than unbelievable $(.51)$ conclusions $\left(F=61.4, M S_{\mathrm{e}}=\right.$ $0.11)$. Also, the interaction was significant $(F=31.2$, $M S_{\mathrm{e}}=0.11$ ), so that the effect of validity was larger for unbelievable (.47) than for believable (.14) conclusions.

The analysis of the RT data likewise revealed two main effects and an interaction (all $p s \leq .021){ }^{2}$ In this case, however, the findings contradicted the disconfirmation hypothesis, which would predict that the reasoners would take longer to process unbelievable conclusions than believable ones. Instead, the reasoners took significantly longer to process believable conclusions $(21.4 \mathrm{sec})$ than to process unbelievable ones $(20.0 \mathrm{sec} ; F=5.42$, $\left.M S_{\mathrm{e}}=45.7\right)$. This finding seems puzzling in light of the acceptance data, which supported the disconfirmation view: The fact that the effect of validity was larger for unbelievable conclusions than for believable ones is consistent with the assumption that a search for alternatives is more likely to occur when reasoners are presented with unbelievable conclusions.

A second unexpected finding concerned the effect of validity. Contrary to prediction, the reasoners also took longer to process invalid conclusions $(23.3 \mathrm{sec})$ than valid ones (18.2 sec; $\left.F=49.1, M S_{\mathrm{e}}=68.4\right)$. This finding is inconsistent with the prediction derived from MMT: Because the discovery of a single, disconfirming model should suffice to reject invalid conclusions, they should have been processed more quickly than valid conclusions, the acceptance of which should require reasoners to test multiple models. Instead, invalid conclusions required longer to process, suggesting that these problems required more effort or processing stages than did the valid ones. Finally, believability and validity interacted ( $\left.F=15.9, M S_{\mathrm{e}}=40.4\right)$, such that believable conclusions took longer to process than unbelievable ones for invalid problems [3.63 sec; $t(127)=3.73, p<.001]$, but not for valid problems $[-0.85 \mathrm{sec} ; t(1,127)=1.35, p>.1]$.

\section{Diagram Drawing}

A diagram was scored as correct if (1) it integrated the $\mathrm{A}, \mathrm{B}$, and $\mathrm{C}$ terms in a manner consistent with the premises and (2) it did not duplicate a previously drawn diagram. The mean number of correct diagrams in each belief by validity cell is represented in the right panel of Figure 1 (because the no-diagram group from Study 1 was not included, the $n$ for this analysis was 96). As is apparent from the figure, none of the main effects or interactions was significant $(p>.1$; this analysis had $80 \%$ power to find a difference of .10 between conditions). On average, people produced about one diagram per problem, regardless of the believability of the conclusion. This is inconsistent with the disconfirmation hypothesis; according to the latter, people should consider more alternative models for unbelievable conclusions than for believable ones.

\section{Individual Differences in Diagram Drawing}

Although most reasoners may consider only a single model of the premises, the willingness to consider alternatives may be a matter of individual differences (Newstead et al., 2002; Torrens et al., 1999). For each participant, we computed three scores: the total number of diagrams drawn, a belief index (number of believable number of unbelievable conclusions accepted), and a validity index (number of valid - number of invalid conclusions accepted). Consistent with past findings (Torrens et al., 1999), individual differences in the number of alternative representations of the premises (i.e., diagrams) was related to individual differences in the belief bias effect and in reasoning ability, as evidenced by the correlation between the total number of diagrams drawn and the belief and validity indices, respectively ( $r \mathrm{~s}=$ -.20 and $.41, p \mathrm{~s}=.028$ and $.001 ; n=96)$. These correlations presumably arise because people who consider alternative models can identify the validity of valid but unbelievable problems and can reject the invalid believable problems.

\section{DISCUSSION}

Our data were inconsistent with two key predictions of the disconfirmation hypothesis: Reasoners spent less time reasoning about unbelievable conclusions than about believable ones and constructed the same number of premise representations when confronted with unbelievable and believable conclusions. These findings are clearly at odds with the assumption that they analyze unbelievable conclusions more, in an attempt to disconfirm them. In contrast, individual differences in the ability to construct alternative representations of premise information predicted both overall performance and the belief bias effect, suggesting that the ability to consider disconfirming possibilities may differentiate good and poor reasoners (Newstead et al., 2002; Torrens et al., 1999). Thus, although our data indicate that the disconfirmation hypothesis does not provide a good account of how reasoners evaluate conclusions, it is possible that the process of disconfirmation may be part of a more general ability associated with sound logical reasoning skills.

Of course, the acceptability of this conclusion hinges on the degree to which one can assume that the only difference between the processing of believable and unbelievable conclusions lies in the putative disconfirmation stage. At least in terms of MMT, the most widely accepted version of the disconfirmation hypothesis, this is clearly the case. The effects of belief are assumed to occur after an initial attempt at reasoning (JohnsonLaird \& Byrne, 1991); that is, regardless of whether the conclusion is believable or unbelievable, reasoners are thought to construct a model of the premises and to evaluate that model against the conclusion provided (Evans et al., 1993). It is at that point that the effect of believability occurs; if the conclusion is unbelievable, a 
search is engaged in to find an alternative model. Thus, the process of reasoning about believable and unbelievable conclusions is explicitly assumed to be identical, except for the disconfirmation stage. Thus, the fact that reasoners take longer to process believable conclusions cannot be accommodated by this view without major modification.

\section{Other Theories of Belief Bias in Deductive Reasoning}

In addition to being broadly inconsistent with the disconfirmation hypothesis, these data are not easily explicable on the basis of current alternative views either. The most comprehensive alternative view of the belief bias effect was proposed by Klauer et al. (2000). Although the mechanisms proposed differ greatly from those proposed by MMT, this view also makes the prediction that unbelievable conclusions will take longer to process than believable ones. According to Klauer et al.'s multinomial model, reasoners evaluate unbelievable conclusions by applying the principle of reductio ad absurdum. One begins by supposing that the logical opposite of the conclusion is true and then attempts to construct a model in which the premises entail this negated conclusion. If such an attempt succeeds, one can reject the original conclusion as invalid; if it fails, there is tentative support for the validity of the conclusion. Since the process of making suppositions and drawing inconsistencies are additional steps that are required in the processing of unbelievable conclusions, but not of believable ones, these conclusions should take longer to process.

The verbal reasoning theory (hereafter, VRT; Polk \& Newell, 1995) also presents an alternative account of the belief bias effect. This theory emphasizes the role played by linguistic processes of encoding and comprehension and downplays the search for alternative models. The goal of encoding processes is to formulate a model similar to the initial model described by MMT. After an initial encoding attempt, reasoners are posited to test the conclusion provided. If the conclusion is consistent with the model, it is accepted; if the conclusion is inconsistent with the model, it is rejected. Alternatively, the model may fail to produce a legal or novel conclusion. When the test is thus inconclusive, the reasoner either may attempt to reencode the premises or may give up. When the reasoner gives up, he or she is assumed to choose randomly between rejecting the conclusion and making a judgment based on beliefs.

In terms of conclusion acceptance, VRT can explain the interaction between logic and beliefs by assuming that the believability of the conclusion affects the encoding process. Specifically, reasoners may be more likely to attempt to reencode the premises when the conclusion is unbelievable than when it is believable. However, on this account, one would expect unbelievable conclusions to take longer to process than believable conclusions, a prediction that is contradicted by our data.

\section{Modified Verbal Reasoning Theory}

We propose a hybrid of these two views to account for our findings, which we will refer to as the modified verbal reasoning theory (MVRT). The theory makes the following assumptions.

1. Reasoners typically search for only a single model of the premises (as per Klauer et al., 2000, and Polk \& Newell, 1995), one that integrates the premises and conclusion in a consistent manner (Klauer et al., 2000).

2 . The search continues until a consistent model is found or a response deadline elapses (Polk \& Newell, 1995).

3. If a consistent model is found before the deadline elapses, reasoners accept the conclusion, largely ignoring believability. If a model is not found before the response deadline elapses, reasoners (a) reject the conclusion, largely ignoring believability, or (b) accept or reject the conclusion on the basis of believability (Polk \& Newell, 1995).

In addition, we make two novel assumptions, which explain the interaction between logic and beliefs on both conclusion acceptance and RTs.

4. We assume that search is easier for valid than for invalid conclusions, because all models of the premises are consistent with a valid conclusion, whereas only some models are consistent with an invalid conclusion. Consequently, it will be easier to find a consistent model integrating a valid conclusion with its premises than one integrating an invalid conclusion.

This assumption has several implications. First, because reasoners are more likely to find a consistent model for valid conclusions than for invalid ones, they reason more quickly about valid conclusions than about invalid ones (Assumption 2) and are more likely to accept valid conclusions (Assumption 3 ) ${ }^{3}$ In addition, the effects of belief on conclusion acceptance are expected to be larger for invalid conclusions than for valid conclusions, because reasoners are more likely to reach the response deadline without finding a consistent model (Assumption 3). Thus, they are more likely to base their decisions solely on their beliefs when reasoning about an invalid problem, producing the standard interaction between validity and beliefs.

5. Finally, we assume that the response deadline is longer for believable than for unbelievable conclusions. Given that believable conclusions are more palatable than unbelievable ones, reasoners may try harder to justify acceptance of these conclusions on logical grounds (see also Baron, 1995; Perkins, 1985) and may search longer to find a consistent model.

Overall, therefore, reasoners should take longer to process believable than unbelievable conclusions, and this effect should be larger when the conclusion is invalid. Specifically, for valid conclusions, it is relatively easy to find a consistent model (Assumption 4), so one would be unlikely to reach the deadline regardless of believability; consequently, the effects of beliefs on RT 
should be relatively small. In contrast, when the task is more difficult (i.e., for invalid conclusions), the motivating effects of beliefs should be observed, and reasoners should work longer processing believable conclusions than unbelievable ones.

\section{CONCLUSIONS}

The data presented are inconsistent with the disconfirmation assumption as applied to the conclusion evaluation paradigm. We cannot rule out the possibility, of course, that the process of disconfirmation plays a role in other tasks-for example, when participants must generate, as opposed to evaluate, conclusions. Indeed, there is evidence to suggest that the processes underlying the generation and evaluation of conclusions might be quite different (Madruga, Moreno, Carriedo, \& Gutiérrez, 2000; Markovits \& Nantel, 1989) and that disconfirmation may play a role when participants are asked to generate conclusions (Santamaria, García-Madruga, \& Johnson-Laird, 1998).

We proposed an MVRT of how reasoners evaluate conclusions on deductive reasoning tasks. Specifically, we proposed that reasoners treat the provided conclusion as part of the problem space and attempt to integrate that conclusion with the premise information. On this task, beliefs may serve in a confirmatory role, rather than in a disconfirmatory one, in that reasoners may persevere longer to find a way to integrate believable conclusions, as compared with unbelievable conclusions.

\section{REFERENCES}

BARON, J. (1995). Myside bias in thinking about abortion. Thinking \& Reasoning, 1, 221-235.

Edwards, K., \& Smith, E. E. (1996). A disconfirmation bias in the evaluation of arguments. Journal of Personality \& Social Psychology, 71, 5-24.

Evans, J. S. B. T., Barston, J. L., \& Pollard, P. (1983). On the conflict between logic and belief in syllogistic reasoning. Memory \& Cognition, 11, 295-306.

Evans, J. S. B. T., Newstead, S. E., Allen, J. L., \& Pollard, P. (1994). Debiasing by instruction: The case of belief bias. European Journal of Cognitive Psychology, 6, 263-285.

Evans, J. S. B. T., Newstead, S. E., \& Byrne, R. M. J. (1993). Human reasoning: The psychology of deduction. Hove, U.K.: Erlbaum.

Johnson-Laird, P. N., \& Byrne, R. (1991). Deduction. Hove, U.K.: Erlbaum

Klauer, K. C., Musch, J., \& Naumer, B. (2000). On belief bias in syllogistic reasoning. Psychological Review, 107, 852-884.

KoeHler, J. J. (1993). The influence of prior beliefs on scientific judgments of evidence quality. Organizational Behaviour \& Human Decision Processes, 56, 28-55.

Madruga, J. A. G., Moreno, S., Carriedo, N., \& Gutiérrez, F. (2000). Task, premise order, and strategies in Rip's conjunction- disjunction and conditionals problems. In W. Shaeken, G. De Vooght, A. Vandierendonck, \& G. d'Ydewalle (Eds.), Deductive reasoning and strategies (pp. 49-71). Mahwah, NJ: Erlbaum.

Markovits, H., \& NAntel, G. (1989). The belief-bias effect in the production and evaluation of logical conclusions. Memory \& Cognition, 17, 11-17.

Newstead, S. E., Handley, S. J., \& Buck, E. (1999). Falsifying mental models: Testing the predictions of theories of syllogistic reasoning. Memory \& Cognition, 27, 344-354.

Newstead, S. E., Pollard, P., Evans, J. S. B. T., \& Allen, J. L. (1992). The source of belief bias effects in syllogistic reasoning. Cognition, 45, 257-284.

Newstead, S. E., Thompson, V. A., \& Handley, S. J. (2002). Generating alternatives: A key component in human reasoning? Memory \& Cognition, 30, 129-137.

OAKHILl, J., \& Johnson-Laird, P. N. (1985). The effects of belief on the spontaneous production of syllogistic conclusions. Quarterly Journal of Experimental Psychology, 37, 553-569.

OAKhill, J., Johnson-Laird, P. N., \& Garnham, A. (1989). Believability and syllogistic reasoning. Cognition, 31, 117-140.

Perkins, D. N. (1985). Post-primary education has little impact on informal reasoning. Journal of Educational Psychology, 77, 562-571.

Polk, T. A., \& Newell, A. (1995). Deduction as verbal reasoning. Psychological Review, 102, 533-566.

Santamaria, C., García-Madruga, J. A., \& Johnson-Laird, P. N. (1998). Reasoning from double conditionals: The effects of logical structure and believability. Thinking \& Reasoning, 4, 97-122.

Stanovich, K. E., \& West, R. F. (1997). Reasoning independently of prior belief and individual differences in actively open-minded thinking. Journal of Educational Psychology, 89, 342-357.

Thompson, V. A. (1996). Reasoning from false premises: The role of soundness in making logical deductions. Canadian Journal of Experimental Psychology, 50, 315-319.

Torrens, D., Thompson, V. A., \& Cramer, K. M. (1999). Individual difference and the belief bias effect: Mental models, logical necessity, and abstract reasoning. Thinking \& Reasoning, 5, 1-28.

\section{NOTES}

1. To confirm parity across groups, three-factor mixed ANOVAs were computed to examine the effects of validity (within subjects), belief (within subjects), and group (between subjects) on RTs, acceptances, and diagrams. None of the main effects or interactions involving the group factor was significant ( $p \geq .07$ ), suggesting that the effects of interest were comparable across groups. Moreover, an analysis that included only data from the no-diagram condition produced a pattern identical to that from the omnibus analysis.

2 . The RT data reported were collapsed over correct and incorrect responses. The pattern is identical when only correct responses are analyzed.

3. Note that this analysis applies only to indeterminately invalid conclusions - that is, conclusions that are consistent with at least one, but not all, models of the premises. In contrast, performance for determinately invalid conclusions (i.e., those that are not consistent with any models of the premises) may entail a different set of processes. In the absence of concrete data, we decided not to speculate as to what these processes might entail and have, instead, limited our discussion to the types of conclusions examined in the present experiment.

(Manuscript received June 26, 2001; revision accepted for publication February 25, 2002.) 